\title{
Entanglement dynamics of electron-electron scattering in low-dimensional semiconductor systems
}

\author{
F. Buscemi, ${ }^{1,2, \mid *}$ P. Bordone, ${ }^{1,2}$ and A. Bertoni ${ }^{1}$ \\ ${ }^{1}$ CNR-INFM, S3 Research Center, Via Campi 213/A, I-Modena 41100, Italy \\ ${ }^{2}$ Dipartimento di Fisica, Università di Modena e Reggio Emilia, Modena, Italy
}

We perform the quantitative evaluation of the entanglement dynamics in scattering events between two insistinguishable electrons interacting via Coulomb potential in $1 \mathrm{D}$ and $2 \mathrm{D}$ semiconductor nanostructures. We apply a criterion based on the von Neumann entropy and the Schmidt decomposition of the global state vector suitable for systems of identical particles. From the time-dependent numerical solution of the two-particle wavefunction of the scattering carriers we compute their entanglement evolution for different spin configurations: two electrons with the same spin, with different spin, singlet, and triplet spin state. The procedure allows to evaluate the mechanisms that govern entanglement creation and their connection with the characteristic physical parameters and initial conditions of the system. The cases in which the evolution of entanglement is similar to the one obtained for distinguishable particles are discussed.

PACS numbers: 03.65.Ud, 03.67.Mn, 73.23.Ad

\section{INTRODUCTION}

Quantum entanglement has undoubtly represented one of the most peculiar aspects of quantum mechanics as it can be viewed as the furthest departure of quantum world from the classical one [1, 2, 3]. Furthermore, in the last years, entanglement has been recognized as the resource for quantum information processing [4, 5]. In this context a growing interest in continuous-variables entanglement is arising for various physical phenomena, such as binary collisions between unbound or trapped particles, photoionization of atoms in both weak

*Electronic address: buscemi.fabrizio@unimore.it 
and strong fields, and photodissociation of molecules, where the entangled subsystems are spatially separated [6, 7, 8, 9]. For binary collisions a particular attention has been devoted to the dynamical creation of entanglement between the two particles, stemming from the possibility of using such a system to investigate the foundations of quantum mechanics itself: it has been shown that an almost maximal violation of Bell's inequality can occur [10]. The quest for quantum-computing capable devices has also produced great interest in entanglement formation in solid state systems where decoherence effects, mainly due to carrier-carrier scattering, play a dominant role [11, 12, 13]. In particular these effects have been studied in the context of electron transport in semiconductors, where the time evolution of the entanglement of spatial degrees of freedom has been calculated numerically for scattering events between two distinguishable charged particles by means of the von Neumann entropy of the density matrix reduced on the spatial degrees of freedom of one of the particles [11, 12]. It should be noticed that the distinguishability of the two particles greatly simplifies the system modelling and that it is representative of only few of the collision events taking place in semiconductors, as an electron-hole scattering. Indeed the notion of entanglement for quantum systems composed of distinguishable particles has been widely investigated from the theoretical and experimental point of view [8, 14], while relevant questions arise for systems of identical particles (both bosons and fermions) 15, 16, 17, 18, 19]. Here, in particular, unlike the distinguishable particles case, the Hilbert space of the physical states does not have a naturally selected structure of tensor product, because of the symmetrization postulate. For example, in the analysis of a scattering event between particles, this implies that the system wavefunction can never be factorized in the product of two one-particle wavefunctions. This makes difficult to give a straightforward definition of entanglement because of the presence of the correlations due to the exchange symmetry.

Only recently the problem has been deeply analyzed for fermion systems. Various approaches have been proposed in literature [17, 18, 19, 20, 21, 22], each of one having its own advantages and drawbacks, and no consensus has been reached on which one is the most suitable [23]. Here we follow the one introduced by Schliemann [18, 24] and recently reexamined and extended by other authors [20, 25, 26], since it allows to use a density matrix in the spatial coordinates and to compute the von Neumann entropy by means of a generalization of the numerical procedure already developed for the case of distinguishable particles. Such an approach is based on an analogous of the Schmidt decomposition for state vectors of 
two fermionic particles: through an unitary transformation the antisymmetric wavefunction is expressed into a basis of Slater determinants with a minimum number of non-vanishing terms. This number, known as Slater rank, is a criterion to identify whether a system is entangled or not, which involves the evaluation of the von Neumann entropy of the oneparticle reduced density matrix. In this notation the von Neumann entropy becomes not only a measure of the uncertainty due to the impossibility of attributing a definite state to each particle of the couple but also of the amount of uncertainty deriving from the indistinguishability of particles. However it is well known that the quantum correlations related to exchange simmetry cannot be used to violate Bell's inequality and are not a resource for quantum information processing [25]: they cannot therefore be considered as a manifestation of genuine quantum entanglement.

The basic significance of a quantitative evaluation of particle-particle entanglement comes also from the link between quantum correlations and decoherence [1]. The latter is, in fact, a manifestation of the former when one drops the exact quantum description of the whole system, considering a part of it as the environment, thus tracing out its quantum coordinates. In our case one can think of one of the particles as representing the environment. As a consequence the amount of entanglement determined by the proposed simulative approach is a direct measure of the decoherence undergone by a carrier as a consequence of a carriercarrier scattering.

In the present paper we aim at calculating entanglement of both spatial and spin degrees of freedom by studying, via a time-dependent numerical analysis, 1D and 2D models of a scattering event between two electrons in semiconductors. The two particles, explicitly considered as indistinguishable, interact through a Coulomb potential. This approach allows us to overcome the hypothesis of distinguishabilty of the particles used in previous works [11, 12]. In the present case quantum entanglement must be analyzed more carefully in order to single-out the effects of the additional correlation induced by indistinguishability. We also evaluate the role of the spin degrees of freedom. We believe that our results can be a valuable guideline for the use of collision events of identical particles in the quantum information processing based on solid state systems and for the estimation of the decoherence rate induced by carrier-carrier scatterings.

The paper is organized as follows. In Sec. [1] we describe the method used to quantify entanglement in two-fermion systems. In Sec. III we investigate the entanglement dynamics 
for the case of a couple of electrons travelling in two parallel, single-mode, quantum wires (1D-system) and in Sec. IV the same analysis is performed for a scattering event between a free propagating and a bound electron in a single-mode two-dimensional electron gas (2Dsystem). Finally in Sec. $\nabla$ we comment the obtained results and draw conclusions.

\section{ENTANGLEMENT EVALUATION FOR TWO-FERMION SYSTEMS}

Here we shall briefly describe the notion of entanglement between two fermions and the approach used for its evaluation, following the theory formulated by Schliemann [18, 24]. Any pure two-fermion state can be written as:

$$
\left|\Psi_{F}\right\rangle=\sum_{i, j}^{2 N} w_{i j} a_{i}^{\dagger} a_{j}^{\dagger}|0\rangle \quad\left\{a_{i}, a_{j}^{\dagger}\right\}=\delta_{i j}
$$

where $w_{i j}$ are the elements of a complex and antisymmetric $(2 N \times 2 N)$ matrix $W$, and $2 N$ is the number of modes for each single particle. $a_{i}^{\dagger}\left(a_{i}\right)$ is the creation(annihilation) operator of a fermion in the $i$-th mode. The normalization condition is $\operatorname{Tr}\left[W^{\dagger} W\right]=1$. Now we need to introduce the fermionic analog of the Schmidt decomposition [28] indicating that exists a unitary transformation $U$ such that

$$
W=U Z U^{T}
$$

where

$$
Z=\operatorname{diag}\left[Z_{1} \ldots Z_{i} \ldots Z_{N}\right]
$$

is a block diagonal matrix with bidimensional blocks $Z_{i}$ of the type:

$$
\mathrm{Z}_{i}=\left(\begin{array}{cc}
0 & z_{i} \\
-z_{i} & 0
\end{array}\right)
$$

We note that $z_{i}$ may also be zero. This decomposition is unique and we can write

$$
\left|\Psi_{F}\right\rangle=\sum_{i}^{N} z_{i}\left(a_{2 i-1}^{\prime \dagger} a_{2 i}^{\prime \dagger}-a_{2 i}^{\prime \dagger} a_{2 i-1}^{\prime \dagger}\right)|0\rangle
$$

where the fermionic state is given in terms of new creation operators defined from $a_{i}^{\dagger}=$ $\sum_{j} u_{i j}^{*} a_{j}^{\prime \dagger}$. In other words one can think of the unitary transformation $U$ as acting on the fermionic operator $a_{i}^{\dagger}$. The number of $z_{i}$ different from zero is the Slater rank and is closely 
related to the entanglement: the state $\left|\Psi_{F}\right\rangle$ is considered non entangled if its Slater rank is equal to 1, while it must be considered a genuinly entangled state if its Slater number is greater than 1. In fact it can be easily seen from Eq. (4) that only in the first case $\left|\Psi_{F}\right\rangle$ can be obtained by antisymmetrization of the product of the single-particle states.

It has been shown recently that the von Neumman entropy, commonly used in the context of non-identical particles, is still a good quantum-correlation measure for identical particles [25, 29]. From the two-particle density matrix $\rho_{F}=\left|\Psi_{F}\right\rangle\left\langle\Psi_{F}\right|$, we can get the one-particle reduced density matrix (normalized to unity) [20]

$$
\rho_{\mu \nu}^{f}=\frac{\operatorname{Tr}\left[\rho_{F} a_{\nu}^{\dagger} a_{\mu}\right]}{\operatorname{Tr}\left[\rho_{F} \sum_{\mu} a_{\mu}^{\dagger} a_{\mu}\right]}=\left(W^{\dagger} W\right)_{\nu, \mu} .
$$

In terms of the coefficients $z_{i}$ the normalization condition becomes $\sum_{i=1}^{N}\left|z_{i}\right|^{2}=1 / 2$, while the von Neumman entropy $\varepsilon$ can be computed from:

$$
\varepsilon=-\operatorname{Tr}\left[\rho^{f} \ln \rho^{f}\right]=\ln 2-2 \sum_{i=1}^{N}\left|z_{i}\right|^{2} \ln 2\left|z_{i}\right|^{2}
$$

where, as stated before, $\left|z_{i}\right|^{2}$ are the eingenvalues of the matrix $\rho^{f}$. For identical particles the von Neumann entropy reaches its minimum value $\varepsilon=\ln 2$ in the case of non entangled states, whose Slater rank is 1, while, for distinguishable particles, the minimum value of the entropy, still corresponding to non entangled states, is zero. This behavior can be explained if we consider the real meaning of the von Neumman entropy, that must be interpreted as a measure of the lack of knowledge about the state of a quantum system. The value ln 2 for non entangled two-fermion states measures the uncertainty arising from the indistinguishability of the particles; in this case the correlations are only related to exchange simmetry and cannot be used as a resource for quantum information. On the other side when the von Neumann entropy is greater than $\ln 2$ there is an additional ignorance about the state of one of the two particles, stemming from genuine entanglement.

\section{1D-SYSTEM}

\section{A. The Theoretical Model}

The physical system consists of two electrons running in opposite directions along two parallel silicon quantum wires. The dynamics of the two particles is 1D and is coupled 
through Coulomb potential, so that the Hamiltonian of the system takes the form

$$
H\left(r_{a}, r_{b}\right)=-\frac{\hbar^{2}}{2 m}\left(\frac{\partial^{2}}{\partial r_{a}^{2}}+\frac{\partial^{2}}{\partial r_{b}^{2}}\right)+V\left(r_{a}, r_{b}\right)
$$

where $V\left(r_{a}, r_{b}\right)$ can be expressed as

$$
V\left(r_{a}, r_{b}\right)=\frac{e^{2}}{\epsilon \sqrt{\left(x_{a}-x_{b}\right)^{2}+d^{2}}}
$$

where $e$ is the electron charge, $\epsilon$ is the silicon dielectric constant, $x_{a}$ and $x_{b}$ the electron coordinates along the wires and $d$ the distance in the perpendicular direction $y$ between the two wires. We use the approximation of single parabolic band and do not consider spin-orbit interaction.

The two quantum wires have been considered as 1D-systems. Each of the two particles has another degree of freedom, besides the spin and space one, indicating the quantum wire in which it is moving, i.e. we suppose that each carrier is confined along the $y$ direction in a wire (see Fig. 11). Moreover each of the two carriers is represented at initial time $t_{0}$ by a

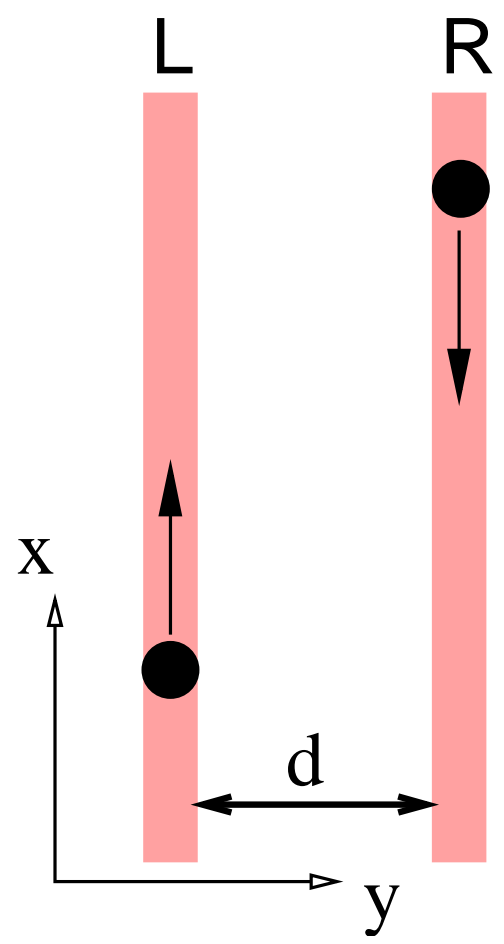

FIG. 1: (Color online) The 1D physical system consisting of two parallel quantum wires, where two electrons run in opposite directions; $d$ is the distance between the wires. 
minimum uncertainty wave packet, so that its space wavefunction takes the form

$$
\psi\left(x, t_{0}\right)=\frac{1}{(\sqrt{2 \pi} \sigma)^{1 / 2}} \exp \left(-\frac{\left(x-x_{0}\right)^{2}}{4(\sigma)^{2}}+i k \cdot x\right)
$$

where $\sigma$ is the mean dispertion in position, $k=\sqrt{2 m E_{k}} / \hbar$ with $m$ the effective mass of the carrier and $E_{k}$ its kinetic energy.

Taking into account both spin and "wire" degrees of freedom, we can consider different initial state configurations. The first quantum state considered is the one having two particles with the same spin (spin up):

$$
|\Psi\rangle=\frac{1}{\sqrt{2}}(|\psi \phi\rangle|\mathrm{LR}\rangle-|\phi \psi\rangle|\mathrm{R} \mathrm{L}\rangle)|\uparrow \uparrow\rangle
$$

where both the wavefunctions corresponding to the states $|\psi\rangle$ and $|\phi\rangle$ are of the type defined in Eq. (91), and the variances of the wavepackets and the distance between their centers are such that the Coulomb energy of the system is negligible at initial time. In Eq. (10), the ket $|L\rangle(|R\rangle)$ indicates that the particle is localized in left (right) wire, and $|\uparrow\rangle$ indicates spin up state.

In order to compute the entanglement as given by Eq. (6) we need to evaluate numerically the quantum state of Eq. (10). To this aim the explicit form of the matrix $W$ has to be obtained. This is done in the Appendix . where we show that $W$ for the same-spin configuration takes the form

$$
\mathrm{W}_{\Psi}=\frac{1}{2 \sqrt{2}}\left(\begin{array}{cccc}
W_{A} & -W_{S} & 0 & 0 \\
W_{S} & -W_{A} & 0 & 0 \\
0 & 0 & 0 & 0 \\
0 & 0 & 0 & 0
\end{array}\right)
$$

where $W_{A}$ and $W_{S}$ are the antisymmetric and symmetric matrices defined in the Appendix

The second quantum state considered is the one of two electrons having different spins. It is not factorizable in a part containing only space and wire variables and in a part containing only spin variables, and reads

$$
|\Upsilon\rangle=\frac{1}{\sqrt{2}}(|\psi \phi\rangle|\mathrm{LR}\rangle|\uparrow \downarrow\rangle-|\phi \psi\rangle|\mathrm{RL}\rangle|\downarrow \uparrow\rangle) .
$$

It should be noticed that for such a state it is possible to make precise claims about the spin of a particle whose position-wire state is known. From the calculations presented in the 
Appendix , we get

$$
\mathrm{W}_{\Upsilon}=\frac{1}{4 \sqrt{2}}\left(\begin{array}{cccc}
W_{A} & -W_{S} & -W_{S} & W_{A} \\
W_{S} & -W_{A} & -W_{A} & W_{S} \\
W_{S} & -W_{A} & -W_{A} & W_{S} \\
W_{A} & -W_{S} & -W_{S} & W_{A}
\end{array}\right) .
$$

The last two states considered still describe two electrons with different spins, but they can be factorized in a position-wire coordinate term and in a spin term. We can identify the singlet spin state

$$
|\Phi\rangle=\frac{1}{2}(|\psi \phi\rangle|\mathrm{LR}\rangle+|\phi \psi\rangle|\mathrm{R} \mathrm{L}\rangle)(|\uparrow \downarrow\rangle-|\downarrow \uparrow\rangle)
$$

with

$$
\mathrm{W}_{\Phi}=\frac{1}{4}\left(\begin{array}{cccc}
0 & 0 & -W_{S} & W_{A} \\
0 & 0 & -W_{A} & W_{S} \\
W_{S} & -W_{A} & 0 & 0 \\
W_{A} & -W_{S} & 0 & 0
\end{array}\right)
$$

and the triplet spin state

$$
|\Xi\rangle=\frac{1}{2}(|\psi \phi\rangle|\mathrm{LR}\rangle-|\phi \psi\rangle|\mathrm{R} \mathrm{L}\rangle)(|\uparrow \downarrow\rangle+|\downarrow \uparrow\rangle)
$$

with

$$
\mathrm{W}_{\Xi}=\frac{1}{4}\left(\begin{array}{cccc}
W_{A} & -W_{S} & 0 & 0 \\
W_{S} & -W_{A} & 0 & 0 \\
0 & 0 & -W_{A} & W_{S} \\
0 & 0 & -W_{S} & W_{A}
\end{array}\right) .
$$

The triplet and singlet states have relevant properties that have been taken into account in evaluating the von Neumann entropy. In fact, as shown in the Appendix the von Neumann entropy of $|\Xi\rangle$ and $|\Phi\rangle$ can be obtained by adding $\ln 2$ to the one of the same-spin state $|\Psi\rangle$. This property, due to the fact that such states are factorizable, remains true also during the time evolution since the Hamiltonian does not include spin terms. This implies that at any time $t$ the triplet and singlet can always be factorized in a position-wire term and a spin term. Therefore we can attribute the offset of the entanglement value to the lack of knowledge about the spin state, in the sense that one cannot make precise claims about the spin state of the particle in a given position-wire state $|\psi \mathrm{L}\rangle$ or $|\phi R\rangle$ [25]. 


\section{B. Numerical results}

In our approach we solve numerically the time-dependent Schröndiger equation for the two-particle spatial wavefunction by means of a Crank-Nicholson finite difference scheme. Once the spatial wavefunctions are obtained at each time step, we calculate the matrices $W$ 's related to the different initial spin state configurations and, from these, we obtain the one-particle reduced density matrixes $\rho$ 's. This allows us to evaluate the time evolution of the entanglement between the spatial and spin degrees of freedom of the two electrons in terms of the von Neumann entropy according to the expression given in Eq. (6). To this purpose we need to diagonalize the one-particle reduced density matrix at each time step and this turns out to be the most demanding part of our algorithm from the point of view of the numerical calculation.

Figs. 2] and 3] show that, at initial time, when the two particles are far enough and Coulomb interaction is negligible, the von Neumann entropy for the states $|\Psi\rangle$ and $|\Upsilon\rangle$ is

$\ln 2$ as expected [25, 29]. As indicated by the theoretical criterion described in Sec. [1 this value can be related to the unavoidable correlations due to exchange symmetry and does not imply genuine quantum entanglement. In fact $|\Psi\rangle$ and $|\Upsilon\rangle$ are initially non entangled. The entanglement increases while the two electrons are approaching each other and finally reaches a stationary value once electrons get apart. The final entanglement, which is a function of the distance $d$ between the two wires, is reported in the insets of Figs. [2 and 3. Following this approach it is possible to obtain a quantitative evaluation of the time of entanglement formation in a scattering event: for the studied case such a time is found to be around 1 picosecond. Obviously the dynamics of entanglement formation depends on the physical parameters of the system, as the initial kinetic energy of the electrons and the variance $\sigma$ of their initial wavepacket. This is illustrated in Fig. [4 where $\varepsilon(t)$ is reported for different values of the variance $\sigma$. Increasing the mean dispersion in position leads to an increase in the time at which the stationary value of entanglement is reached, but the value itself is pratically not affected.

We note that the dependence on $d$ of the final values of the entanglement is significantly different for the case of the state $|\Psi\rangle$ with respect to the case of the state $|\Upsilon\rangle$. While for $|\Psi\rangle$ this value decreases monotonically as the distance between the two wires is increased, for $|\Upsilon\rangle$ it shows a well defined maximum (around $d=1 \mathrm{~nm}$ in Fig. [3inset). This means that, in the 


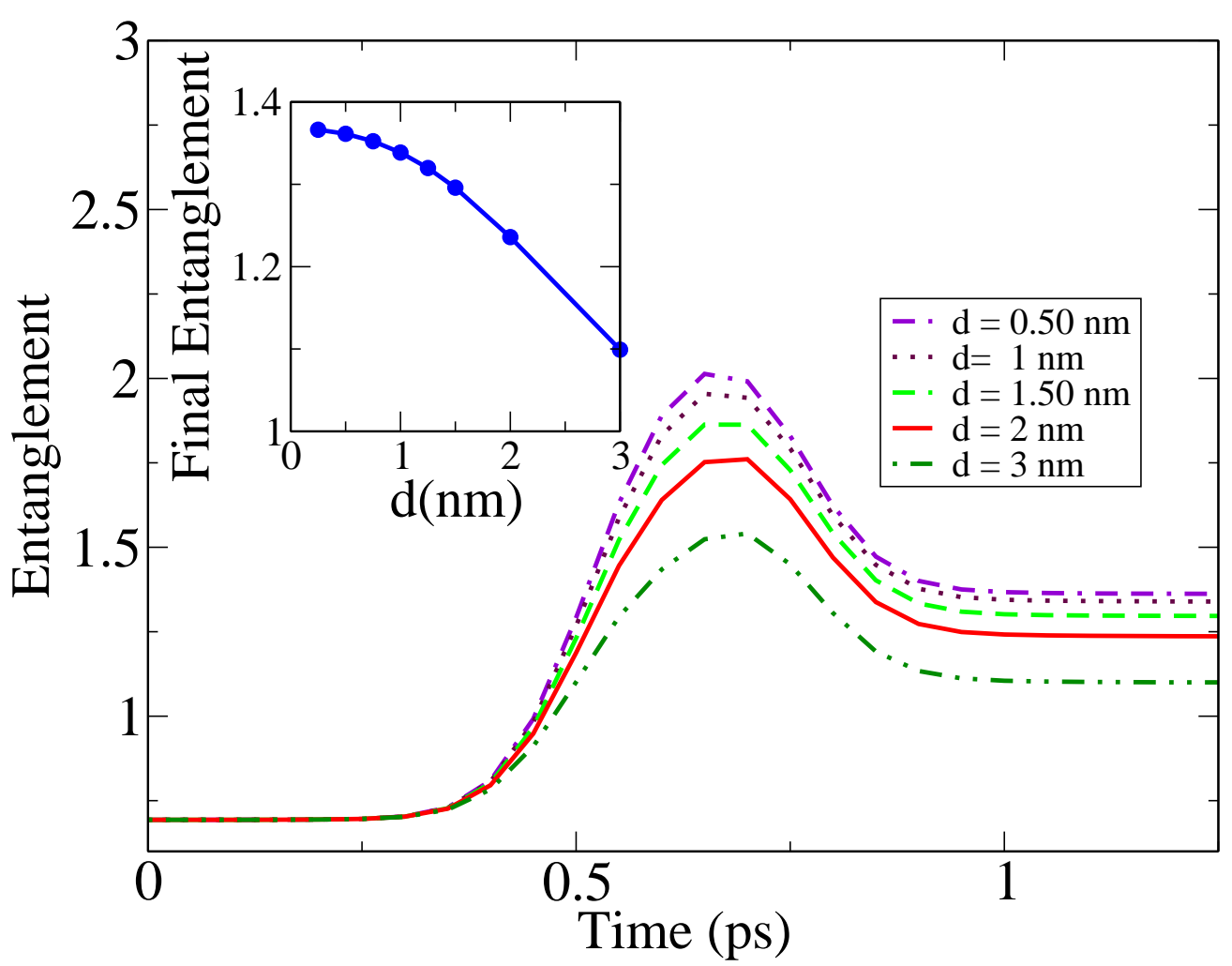

FIG. 2: (Color online) Entanglement as a function of time for the state $|\Psi\rangle$ desribing two electrons with the same spin for different values of the distance $d$ between the two wires. The two electrons have the same initial energy $E_{k}=50 \mathrm{meV}$ and are described by two wavepackets with an initial mean dispersion $\sigma=20 \mathrm{~nm}$. The inset shows the stationary values of the entanglement as a function of $d$.

case of electrons having the same spin, the scattering creates strong quantum correlations between the particles which are weakly affected by the wire distance. On the other hand for electrons with different spins we find a strong dependence on $d$ of the stationary values of the entanglement. This behavior is in good qualitative agreement with the one obtained in previous works in the case of distinguishable particles.

In Fig. 5 we report the time evolution of the entanglement for two values of the distance between the wires and for all the considered spin configurations. Intially the von Neumann entropy of the singlet $|\Phi\rangle$ and triplet $|\Xi\rangle$ states has the value $2 \ln 2$. In agreement with the theoretical predictions its time evolution results to be the same obtained for the same-spin state $|\Phi\rangle$, just shifted by $\ln 2$. Thus at the initial time the singlet and triplet states have to be considered as entangled according to the notion of entanglement for fermionic particles used 


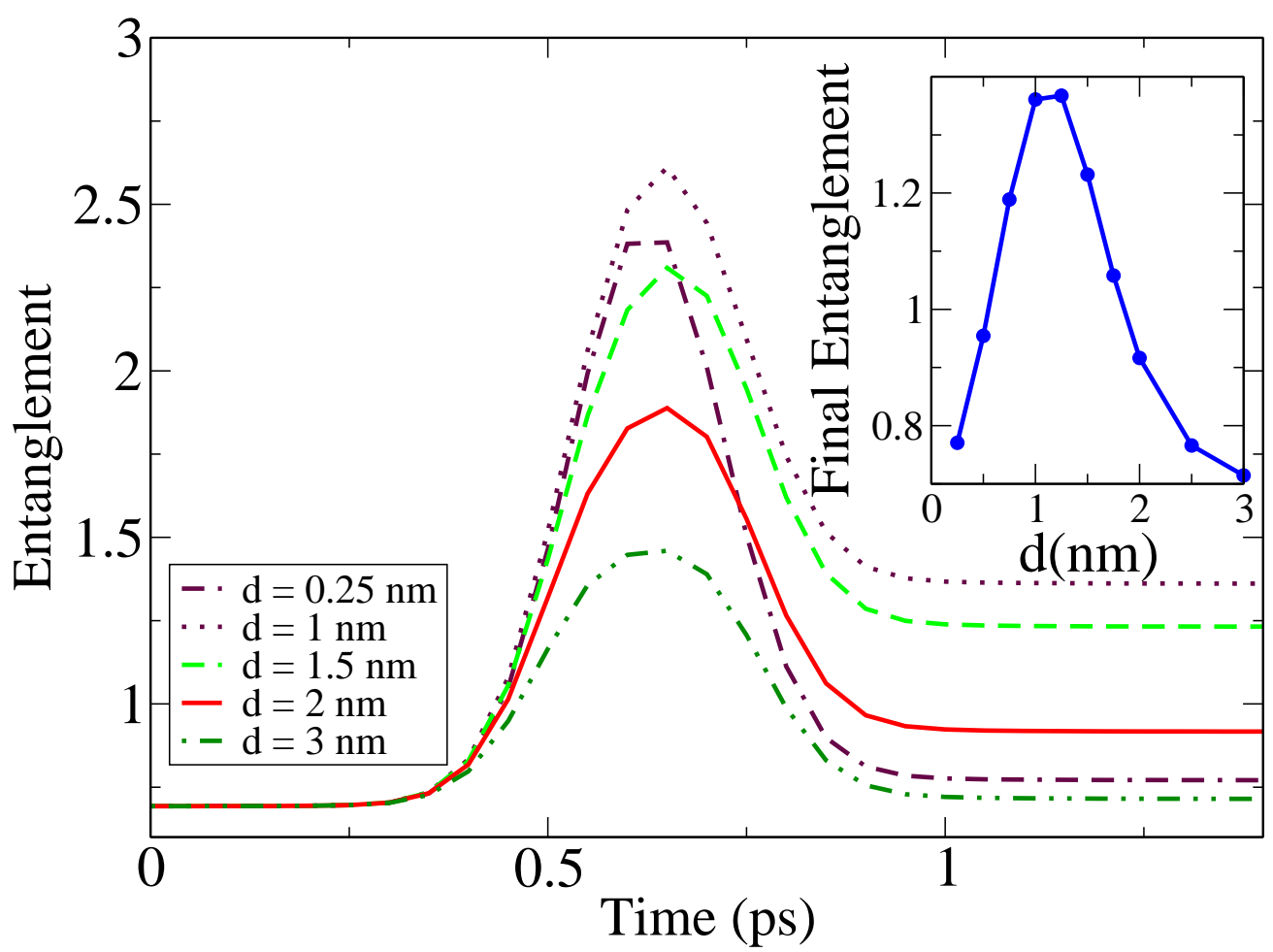

FIG. 3: (Color online) Entanglement as a function of time for the state $|\Upsilon\rangle$ (see Eq. (12)) for different values of the distance $d$ between the two wires. The two electrons have the same initial energy $E_{k}=50 \mathrm{meV}$ and are described by two wavepackets with an initial mean dispersion $\sigma=$ $20 \mathrm{~nm}$. The inset shows the stationary values of the entanglement as a function of $d$.

in this work. The appearance of the offset above mentioned can be explained considering the mathematical form of $|\Phi\rangle$ and $|\Xi\rangle$ as given in Eqs. (14) and (16). Their Slater rank is greater than 1 since they cannot be put in terms of a single Slater determinant: in fact they are not obtained by antisymmetrization of the product of single-particle states. This also confirms that the von Neumann entropy, as a measure of entanglement, corresponds to the Slater rank criterion [20, 25].

\section{2D-SYSTEM}

\section{A. The theoretical model}

We study in this Section the entanglement formation for the case of an electron propagating in a $2 \mathrm{D}$ system and interacting with another electron bound to a specific site by a 


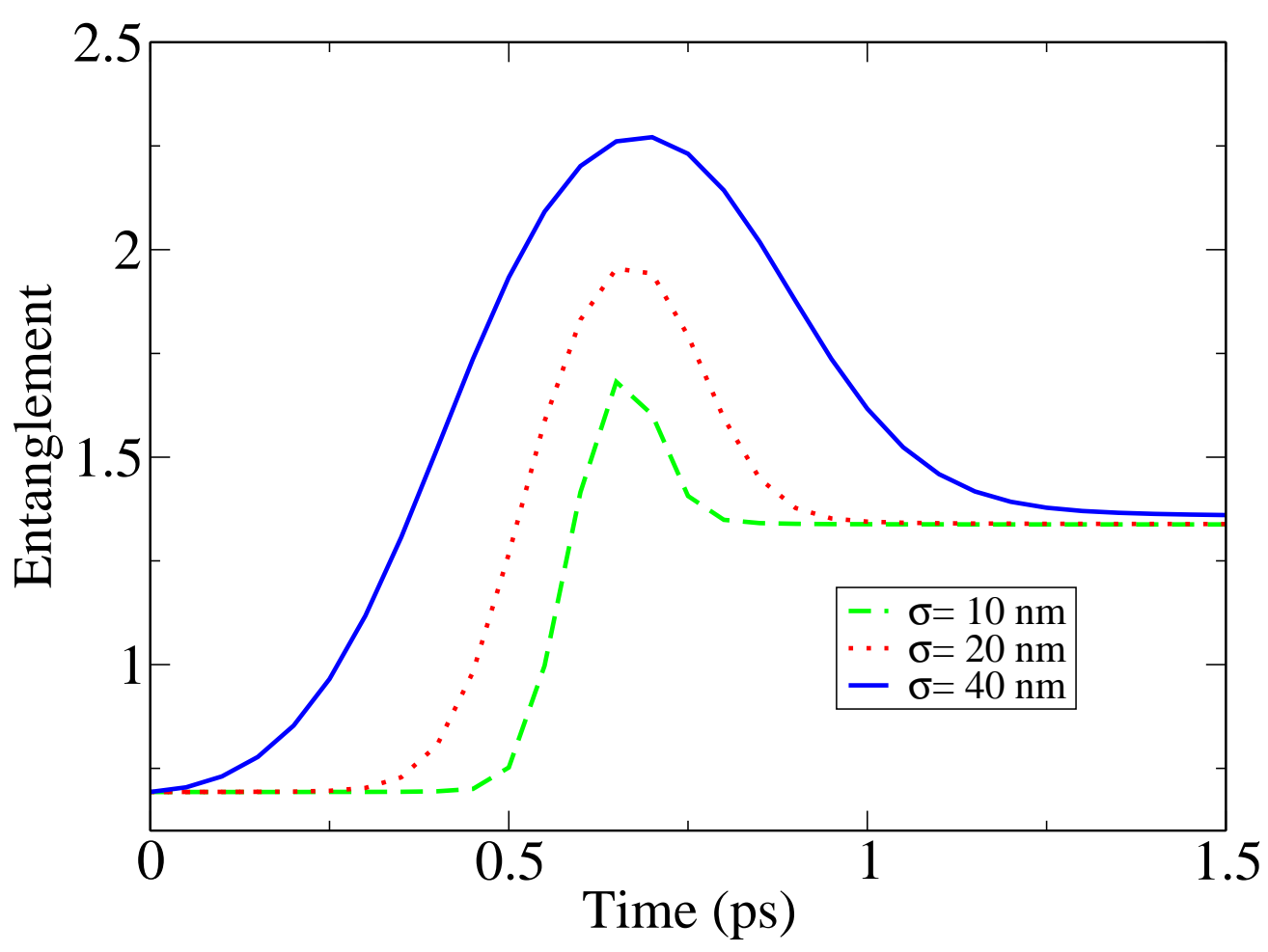

FIG. 4: (Color online) Entanglement as a function of time for the same-spin state $|\Psi\rangle$ at different values of the initial mean dispersion $\sigma$ of the spatial wavefunctions. The initial kinetic energy $E_{k}$ is $50 \mathrm{meV}$ for both electrons. Note that the final stationary value is pratically independent upon $\sigma$.

harmonic potential. The latter can be considered as a simple model of a shallow impurity in a semiconductor. Here the physical parameters of GaAs have been used. The dynamics of the particle bound in the harmonic potential is coupled to the incoming particle through a Coulomb interaction. The Hamiltonian reads

$$
H\left(\mathbf{r}_{a}, \mathbf{r}_{b}\right)=-\frac{\hbar^{2}}{2 m}\left(\frac{\partial^{2}}{\partial \mathbf{r}_{a}^{2}}+\frac{\partial^{2}}{\partial \mathbf{r}_{b}^{2}}\right)+\frac{e^{2}}{\epsilon\left|\mathbf{r}_{a}-\mathbf{r}_{b}\right|}+\frac{1}{2} m \omega^{2}\left(\mathbf{r}_{a}-\mathbf{r}_{0}\right)+\frac{1}{2} m \omega^{2}\left(\mathbf{r}_{b}-\mathbf{r}_{0}\right)
$$

where $\epsilon$ and $m$ are the GaAs dielectric constant and effective mass, respectively, and $\mathbf{r}_{0}$ is the center of the harmonic potential, with energy-level spacing $\hbar \omega$.

At the initial time $t_{0}$ one of the two particles (incoming electron) is represented by a minimum uncertainty wavepacket $\psi\left(\mathbf{r}, t_{0}\right)$ centered in $\mathbf{r}_{1}$, with $\mathbf{k}$ vector pointing towards $\mathbf{r}_{0}$, while the other (bound electron) is in the ground state of the 2D harmonic oscillator

$$
\phi\left(\mathbf{r}, t_{0}\right)=\left(\frac{m \omega}{\pi \hbar}\right)^{1 / 2} \exp \left(-\frac{m \omega\left(\mathbf{r}-\mathbf{r}_{0}\right)^{2}}{2 \hbar}\right)
$$



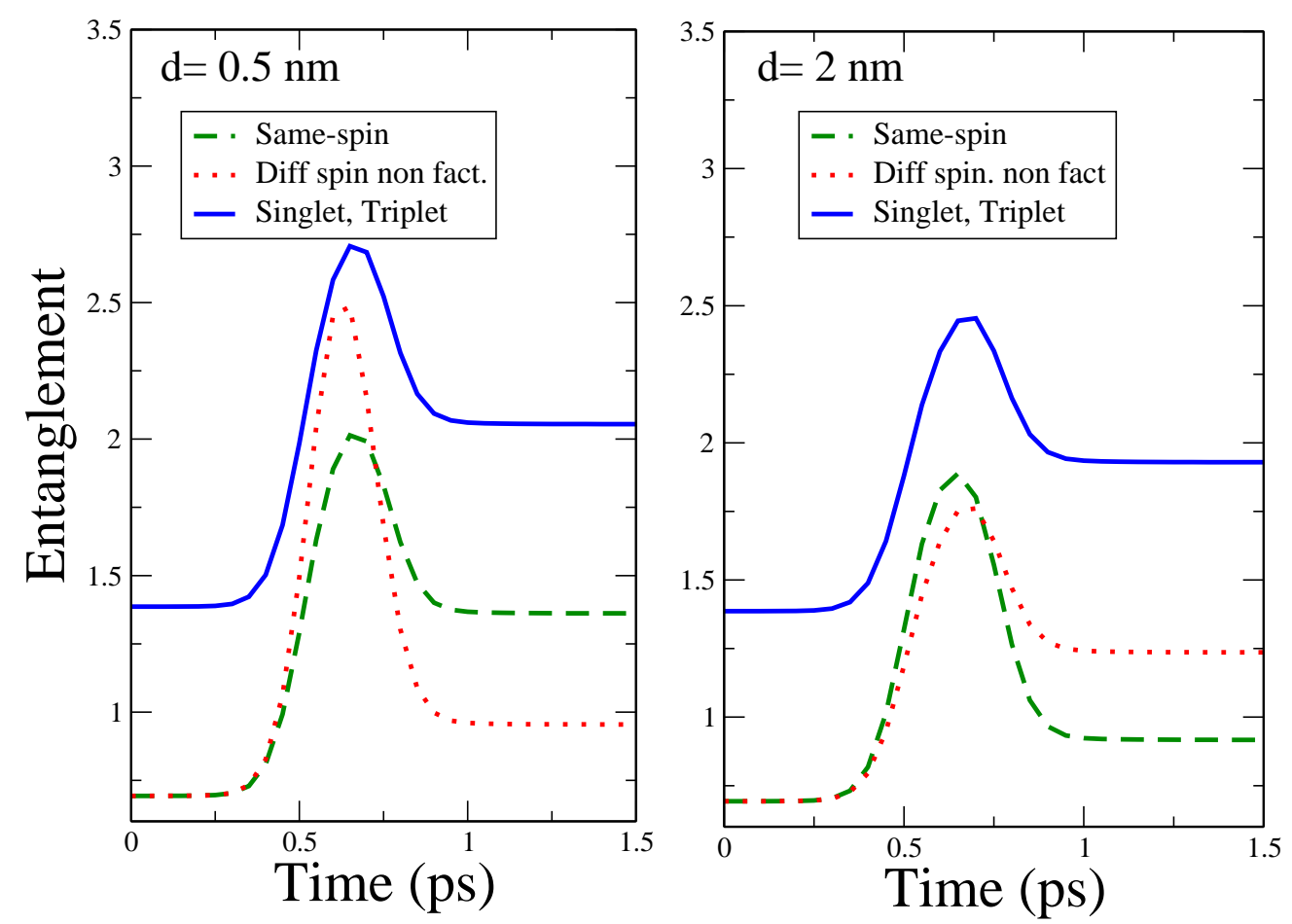

FIG. 5: (Color online) Entanglement as a function of time: two electrons with the same spin $|\Psi\rangle$ (dashed lines), with different spin $|\Upsilon\rangle$ (dotted line), singlet $|\Phi\rangle$ and triplet $|\Xi\rangle$ (solid line). The two wavepackets describing the electrons have both an initial energy $E_{k}=50 \mathrm{meV}$ and initial mean dispersion $\sigma=20 \mathrm{~nm}$. Two different values of the distance $d$ between the two wires are considered: $\mathrm{d}=0.5 \mathrm{~nm}$ in the left panel and $\mathrm{d}=2 \mathrm{~nm}$ in the right panel.

The distance $\left|\mathbf{r}_{1}-\mathbf{r}_{0}\right|$ is such that at the initial time $t_{0}$ the Coulomb energy is negligible. Moreover we have taken $\sigma=\sqrt{\hbar / 2 m \omega}$ : this allowed us to evaluate the time evolution of the two-particle wavefunction in the center-of-mass coordinate system.

Let us now consider the state $|\Psi\rangle$ describing the system with the two particles having the same spin. At the initial time

$$
|\Psi\rangle=\frac{1}{\sqrt{2}}(|\psi \phi\rangle-|\phi \psi\rangle)|\uparrow \uparrow\rangle .
$$

When we discretize each space dimension with a grid of $\mathrm{N}$ points we obtain

$$
|\Psi\rangle=\frac{1}{\sqrt{2}} \sum_{i, j}^{N^{2}}\left(\psi\left(\mathbf{r}_{i}\right) \phi\left(\mathbf{r}_{j}\right)-\phi\left(\mathbf{r}_{i}\right) \psi\left(\mathbf{r}_{j}\right)\right)\left|\mathbf{r}_{i} \mathbf{r}_{j}\right\rangle|\uparrow \uparrow\rangle
$$

and applying the same formalism used for the 1D system of Section III we get for the 
$2 N^{2} \times 2 N^{2}$ matrix $W$ associated to the same-spin state

$$
\mathrm{W}_{\Psi}=\frac{1}{\sqrt{2}}\left(\begin{array}{cc}
W_{A} & 0 \\
0 & 0
\end{array}\right)
$$

where $W_{A}$ is the antisymmetric $N^{2} \times N^{2}$ matrix whose elements read

$$
w_{i j}=\psi\left(\mathbf{r}_{i}\right) \phi\left(\mathbf{r}_{j}\right)-\phi\left(\mathbf{r}_{i}\right) \psi\left(\mathbf{r}_{j}\right)
$$

obtained by the antisymmetrization of the product of the single-particle wavefunctions $\psi$ and $\phi$. The other spin configuration we study in this Section is the triplet spin state

$$
|\Xi\rangle=\frac{1}{2}(|\psi \phi\rangle-|\phi \psi\rangle)(|\uparrow \downarrow\rangle+|\downarrow \uparrow\rangle)
$$

for which

$$
\mathrm{W}_{\Xi}=\frac{1}{2}\left(\begin{array}{cc}
W_{A} & 0 \\
0 & -W_{A}
\end{array}\right) .
$$

Also in this case the von Neumann entropy of the triplet $|\Xi\rangle$ is obtained by the one of the same-spin state $|\Psi\rangle$ adding $\ln 2$. The singlet state and the state with the electrons having different spins and non factorizable into a spin term and a space term have not been considered in the 2D case, since they need a much larger computational effort that is beyond the scope of the present work.

\section{B. Numerical results}

The time evolution of the 2D two-particle wavefunction $|\Psi\rangle$ has been performed in the center-of-mass coordinate system, by means of a finite-difference solution of the corresponding time-dependent Schrödinger equation [30] .

At initial time the centers of the wavepackets are distant enough so that the Coulomb energy is negligible: as a consequence, when the system is described by the state $|\Psi\rangle$ (the two electrons have the same spin), no correlation is present apart from the one due to the exchange symmetry. In fact we see from Fig. [6 that for any value of the energy of the incoming electron the initial von Neumman entropy gets its minimum value $\ln 2$. As the particles get closer their quantum correlation builds up and entanglement reaches a stationary value once particles are far enough. Here the final amount of entanglement 
depends on the initial energy of the carrier: it is higher for higher energies. The time evolution of $\varepsilon(t)$ for the triplet state can be obtained simply shifting by $\ln 2$ the curves reported in Fig. 6]

Fig. [7 is intended to give a better insight into the role played by the correlations between the space degrees of freedom in the entanglement creation, for the $|\Psi\rangle$ state case. To this purpose we have calculated the square modulus of the wavefunction, at two different times, keeping the position of one of the two electrons fixed (indicated by the black spot in the figures). The first time (left column) corresponds to a condition of minimum entanglement, the second (right column) to a condition in which entanglement has already reached its stationary values. We note that when entanglement is at its lowest level the result is independent on the choice of the fixed position while this is not true once entanglement has been created by the Coulomb interaction.

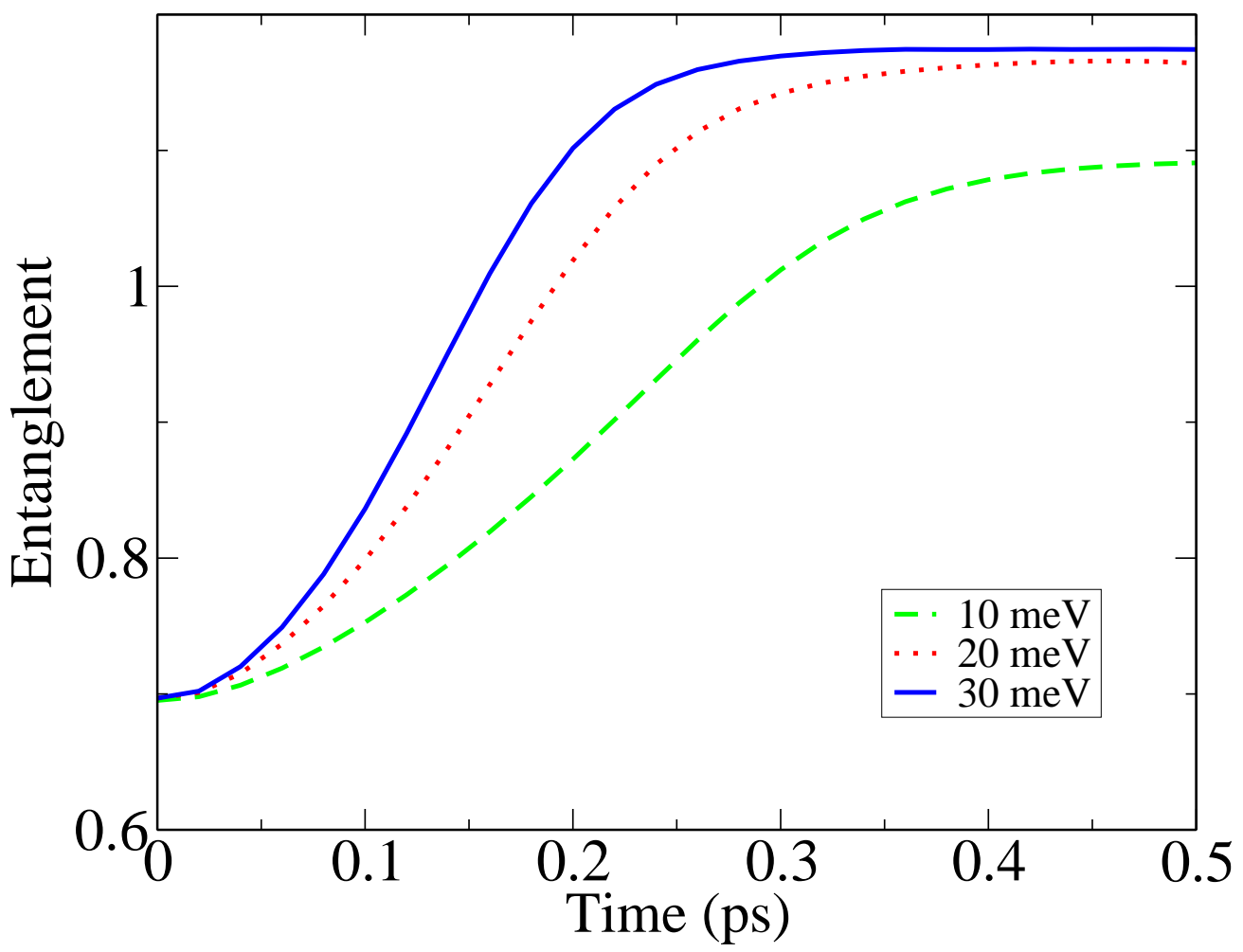

FIG. 6: (Color online) The time evolution of entanglement for $|\Psi\rangle$ (see Eq. (20)) in the 2D system at three different values of the initial energy of the incoming electron, namely $E_{k}=10 \mathrm{meV}$ (dashed line), $20 \mathrm{meV}$ (dotted line), $30 \mathrm{meV}$ (solid line). The harmonic oscillator energy is $\hbar \omega=2 \mathrm{meV}$. 

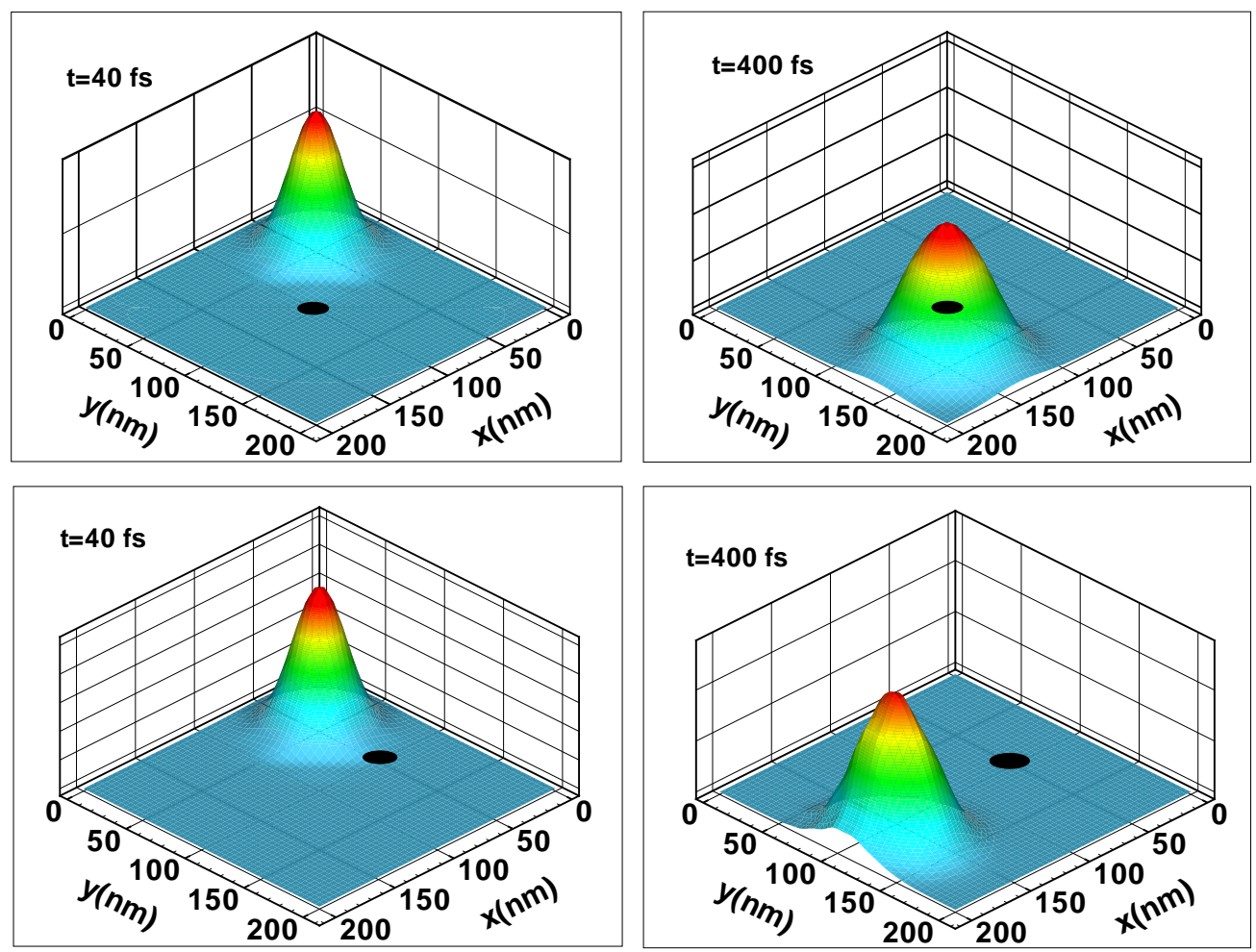

FIG. 7: (Color online) The square modulus of the two-particle antisymmetrized wavefunction $\psi(\mathbf{r}) \phi(\mathbf{R})-\phi(\mathbf{R}) \psi(\mathbf{r})$ for $|\Psi\rangle$ at two different times: $t=40$ fs and $t=400$ fs. In the two upper graphs the position of one of two particles is fixed to $\mathbf{R}=(100 \mathrm{~nm}, 100 \mathrm{~nm})$, while in the two lower graphs $\mathbf{R}=(50 \mathrm{~nm}, 150 \mathrm{~nm})$.

\section{CONCLUSIONS}

In this paper we have studied the dynamics of entanglement formation in systems composed by two indistinguishable particles. To this aim we applied a theoretical method [18, 24], which permits the quantitative evaluation of entanglement for a two-fermion system. The method is based on a fermionic analog of the Schmidt decomposition of the state vector and agrees with von Neumman entropy of the single-particle reduced density matrix, which is a good correlation measure and allows one to determine whether the lack of knowledge is due to a genuine entanglement or only to particles indistinguishabilty. It should be noticed that the correlations due to exchange symmetry cannot be used to implement quantum information processing or violate Bell's inequality [25, 29].

The time-dependent numerical implementation of the theoretical method we developed 
allows one to investigate the decoherence effects that are consequence of carrier scattering, and that can be quantified in terms of entanglement between the scattered particles. This approach makes feasible a direct quantification of the time of the entanglement formation. The numerical results have been obtained using the effective mass of electrons in semiconductors ( $\mathrm{Si}$ for the 1D model and GaAs for the 2D model) but they can be considered as representative of a general behavior.

Our simulations show that the final value of entanglement between two electrons undergoing a scattering event depends only upon some specific physical parameters, as the distance between the quantum wires (in the 1D case), or the initial kinetic energy of the particles, while it is essentially immune to the modifications of other parameters, like the variance $\sigma$ of the initial wavepackets. We showed how the entanglement dynamics depends on the spin components of the state vector describing the system at the initial time, even if the Hamiltonian does not contain spin terms. This suggests the existence of a deep relation between spin states and space entanglement, which implies the possibility of detecting quantum correlations in spin measurements even for the case of wavepackets extending in two distant, not overlapping, regions of space. Moreover, in the 1D system, the numerical results for singlet and triplet spin states shows, at the initial time, quantum correlations related not only to exchange simmetry but also to genuine entanglement. They can be attributed to the lack of knowledge about the spin state of a particle in a specific wire, in agreement with theoretical predictions.

The results obtained in the case of two electrons having different spins are in good qualitative agreement with the case of distinguishable particles [11, 12] apart from the offset term deriving from exchange simmetry. This also confirms that the theoretical framework given in Refs. [18, 20, 25, 26] is a suitable criterion for the evaluation of the entanglement in twofermion systems and is equivalent, in the case of distinguishable particles to the commonly used criterion based on the Schmidt number or on the von Neumman entropy.

\section{Acknowledgments}

We are pleased to thank Carlo Jacoboni for fruitful discussions. We acknowledge support from the U.S Office of Naval Research (contract No. N00014-03-1-0289/ N00014-98-1-0777) and INFM-CNR Progetto Supercalcolo 2006 CINECA. 


\section{APPENDIX: DETERMINATION OF W MATRICES}

Here we illustrate the procedure we apply to the different initial states in order to evaluate numerically the von Neumann entropy in 1D physical systems with each electron localized in the left $(|\mathrm{L}\rangle)$ or right $(|\mathrm{R}\rangle)$ wire, as explained in Sec III. Let us begin with the state $|\Psi\rangle$ defined in Eq. (10) where both particles have spin up but different position-wire wavefunctions described by $|\psi\rangle|\mathrm{L}\rangle$ or $|\phi\rangle|\mathrm{R}\rangle$. We discretize the $x$ coordinate of the electrons along the two wires with a $\mathrm{N}$ points grid: the dimension of each single-particle Hilbert space is $2 N$, taking into account the spin degrees of freedom. In this way we can consider a fermionic position operator $a^{\dagger}\left(x_{l}\right)$ that creates a particle in the point $x_{l}: a^{\dagger}\left(x_{l}\right)|0\rangle=\left|x_{l}\right\rangle$.

We can then express the state $|\Psi\rangle$ as

$$
\begin{aligned}
|\Psi\rangle & =\frac{1}{\sqrt{2}} \sum_{l, m}^{N}\left(\psi\left(x_{l}\right) \phi\left(x_{m}\right)|\mathrm{LR}\rangle-\phi\left(x_{l}\right) \psi\left(x_{m}\right)|\mathrm{RL}\rangle\right) \\
& \times\left|x_{l} x_{m}\right\rangle|\uparrow \uparrow\rangle .
\end{aligned}
$$

We observe that in Eq. (A.1) the state $|\Psi\rangle$ is not antisymmetric with respect to the exchange of the $l$ and $m$ indeces, and, in this form, it cannot be used to evaluate the entanglement according to the theory developed in Sec. [I This difficulty can be overcome by applying a unitary transformation to the wire degrees of freedom:

$$
\begin{aligned}
& \left|V_{1}\right\rangle=1 / \sqrt{2}(|\mathrm{~L}\rangle+|\mathrm{R}\rangle) \\
& \left|V_{2}\right\rangle=1 / \sqrt{2}(|\mathrm{~L}\rangle-|\mathrm{R}\rangle) .
\end{aligned}
$$

Inserting the closure relation in Eq. (A.1), we get

$$
\begin{aligned}
|\Psi\rangle & =\frac{1}{\sqrt{2}} \sum_{l, m}^{N} \sum_{s, t=1}^{2}\left(\psi\left(x_{l}\right) \phi\left(x_{m}\right) \gamma_{\mathrm{L}}(s) \gamma_{\mathrm{R}}(t)-\phi\left(x_{l}\right) \psi\left(x_{m}\right) \gamma_{\mathrm{R}}(s) \gamma_{\mathrm{L}}(t)\right) \\
& \times\left|x_{l} x_{m}\right\rangle\left|V_{s} V_{t}\right\rangle|\uparrow \uparrow\rangle
\end{aligned}
$$

where we introduced $\gamma_{\mathrm{L}}(1)=\gamma_{\mathrm{L}}(2)=\gamma_{\mathrm{R}}(1)=\frac{1}{\sqrt{2}}$ and $\gamma_{\mathrm{R}}(2)=-\frac{1}{\sqrt{2}}$.

Now we can express the state $|\Psi\rangle$ in Eq. (A.3) in terms of fermionic operators $f_{i}^{\dagger}$ defined as:

$$
f_{i}^{\dagger}|0\rangle=\left\{\begin{array}{cc}
\left|x_{i}\right\rangle\left|V_{1}\right\rangle|\uparrow\rangle & \text { for } \quad 1 \leq i \leq N \\
\left|x_{i-N}\right\rangle\left|V_{2}\right\rangle|\uparrow\rangle & \text { for } \quad N+1 \leq i \leq 2 N \\
\left|x_{i-2 N}\right\rangle\left|V_{1}\right\rangle|\downarrow\rangle & \text { for } \quad 2 N+1 \leq i \leq 3 N \\
\left|x_{i-3 N}\right\rangle\left|V_{2}\right\rangle|\downarrow\rangle & \text { for } \quad 3 N+1 \leq i \leq 4 N
\end{array}\right.
$$


and we get

$$
|\Psi\rangle=\sum_{i, j}^{4 N} w_{i j} f_{i}^{\dagger} f_{j}^{\dagger}|0\rangle
$$

where the $4 N \times 4 N$ matrix of the coefficients $w_{i j}$ takes the form of Eq. (11), where $W_{A}$ is an antisymmetric $N \times N$ matrix whose elements read

$$
w_{A_{l m}}=\psi\left(x_{l}\right) \phi\left(x_{m}\right)-\phi\left(x_{l}\right) \psi\left(x_{m}\right)
$$

and $W_{S}$ is its symmetric conterpart. They have been obtained by antisymmetrizing or symmetrizing the product of the single-particle spatial wavefunctions $\phi$ and $\psi$ calculated in the grid points $l$ and $m$.

Now the whole matrix $W_{\Psi}$ is antisymmetric and the state $|\Psi\rangle$ in Eq. (A.4) is expressed in a form that allows us to apply the Schliemann theory. The normalization condition $\operatorname{Tr}\left[W_{\Psi}^{\dagger} W_{\Psi}\right]=1$ is satisfied. Moreover during the time evolution $W_{\Psi}$ mantains this form since the Hamiltonian does not contain spin terms and the coefficients corresponding to the modes where particles have spin down will always be zero.

For the initial state $|\Upsilon\rangle$ of Eq. (12), with two electrons having different spins and not factorizable in a part containing only space and "wire" variables and in a part containing only spin variables, we again discetize the $x$ coordinate into a $N$ points grid and consider the change of basis given in Eq. A.2. In this case we have to apply a unitary transformation also for the spin variables: from $|\uparrow\rangle$ and $|\downarrow\rangle$ to $\left|S_{1}\right\rangle$ and $\left|S_{2}\right\rangle$

$$
\begin{aligned}
& \left|S_{1}\right\rangle=1 / \sqrt{2}(|\uparrow\rangle+|\downarrow\rangle) \\
& \left|S_{2}\right\rangle=1 / \sqrt{2}(|\uparrow\rangle-|\downarrow\rangle) .
\end{aligned}
$$

Now we can express $|\Upsilon\rangle$ as

$$
\begin{array}{r}
|\Upsilon\rangle=\frac{1}{\sqrt{2}} \sum_{l, m}^{N} \sum_{s, t, u, v=1}^{2}\left(\psi\left(x_{l}\right) \phi\left(x_{m}\right) \gamma_{\mathrm{L}}(s) \gamma_{\mathrm{R}}(t) \beta_{\uparrow}(u) \beta_{\downarrow}(v)-\right. \\
\left.\phi\left(x_{l}\right) \psi\left(x_{m}\right) \gamma_{\mathrm{R}}(s) \gamma_{\mathrm{L}}(t) \beta_{\downarrow}(u) \beta_{\uparrow}(v)\right)\left|x_{l} x_{m}\right\rangle\left|V_{s} V_{t}\right\rangle\left|S_{u} S_{v}\right\rangle
\end{array}
$$

where $\beta_{\uparrow}(1)=\beta_{\uparrow}(2)=\beta_{\downarrow}(1)=\frac{1}{\sqrt{2}}$ and $\beta_{\downarrow}(2)=-\frac{1}{\sqrt{2}}$.

If we define the fermionic creation operators $g_{i}^{\dagger}$ as:

$$
g_{i}^{\dagger}|0\rangle=\left\{\begin{array}{cc}
\left|x_{i}\right\rangle\left|V_{1}\right\rangle\left|S_{1}\right\rangle & \text { for } \quad 1 \leq i \leq N \\
\left|x_{i-N}\right\rangle\left|V_{2}\right\rangle\left|S_{1}\right\rangle & \text { for } \quad N+1 \leq i \leq 2 N \\
\left|x_{i-2 N}\right\rangle\left|V_{1}\right\rangle\left|S_{2}\right\rangle & \text { for } \quad 2 N+1 \leq i \leq 3 N \\
\left|x_{i-3 N}\right\rangle\left|V_{2}\right\rangle\left|S_{2}\right\rangle & \text { for } \quad 3 N+1 \leq i \leq 4 N
\end{array}\right.
$$


the state $|\Upsilon\rangle$ in Eq. (12) can be expressed as

$$
|\Upsilon\rangle=\sum_{i, j}^{4 N} w_{i j} g_{i}^{\dagger} g_{j}^{\dagger}|0\rangle
$$

The $4 N \times 4 N$ matrix $W_{\Upsilon}$ reads as in Eq. (13).

Finally we are able to apply to the singlet spin state of Eq. (14) and to triplet spin state of Eq. (16) the same procedure used above for $|\Upsilon\rangle$, and we find that $|\Phi\rangle$ is given in an expression similar to Eq. (A.8), for which the matrix $W$ takes the form of Eq. (15), while for the state $|\Xi\rangle W$ takes the form of Eq. (16). For the sake of brevity we do not report explicitely those calculations.

Now we shall show that the von Neumann entropy $\varepsilon_{\Xi}$ of the triplet $|\Xi\rangle$ and the singlet $|\Phi\rangle$ states (both representing the electrons with opposite spin) can easily be related to the entropy $\varepsilon_{\Psi}$ of the same-spin state $|\Psi\rangle$. Firstly we note that $|\Xi\rangle$ and $|\Phi\rangle$ have the same entropy: this depends on the fact that the one-particle reduced density matrix $W^{\dagger} W$ results to be the same in the two cases. Secondly we observe that the matrix $W_{\Xi}$ can be viewed as a block diagonal matrix with 2 blocks of dimension $2 N$. Therefore for the eingenvalues $\left|z_{i}\right|^{2}$ of $W_{\Xi}^{\dagger} W_{\Xi}$ holds the relation

$$
\left|z_{i}^{\Xi}\right|^{2}=\left|z_{i+2 N}^{\Xi}\right|^{2}
$$

In this case the $\varepsilon_{\Xi}$ can be written as:

$$
\varepsilon_{\Xi}=\ln 2-2 \sum_{i=1}^{2 N}\left|z_{i}^{\Xi}\right|^{2} \ln 2\left|z_{i}^{\Xi}\right|^{2} .
$$

The matrix $W_{\psi}$ has the same upper left block as $W_{\Xi}$ and is zero elsewhere. This implies that the first $2 N$ eingenvalues of the matrix $W_{\psi}^{\dagger} W_{\psi}$ are equal (apart from the constant factor $\frac{1}{2}$ coming from the normalization condition) to the first $2 N$ eingenvalues of the matrix $W_{\Xi}^{\dagger} W_{\Xi}$. Using (A.9) and (A.10) we can relate the von Neumann entropy for $|\Xi\rangle$ to the one for $|\Psi\rangle$

$$
\varepsilon_{\Xi}=\ln 2-4 \sum_{i=1}^{N} \frac{1}{2}\left|z_{i}^{\Psi}\right|^{2} \ln 2 \frac{1}{2}\left|z_{i}^{\Psi}\right|^{2}=\varepsilon_{\Psi}+\ln 2
$$

where we have taken into account the normalization condition $\sum_{i=1}^{N}\left|z_{i}^{\Psi}\right|^{2}=\frac{1}{2}$, recalling $z_{i}^{\Psi}=0$ for $i>2 N$. Eq. (A.11) implies that the von Neumann entropy of the triplet spin 
state can be simply obtained from the one of same-spin state by adding $\ln 2$.

[1] D. Giulini et al., Decohence and the Appearance of a Classical World in Quantum theory (Springer, Berlin, 1996.)

[2] A. Peres, Quantum Theory: Concepts and Methods (Kluwer Academy Publishers, The Netherlands, 1995.)

[3] E. Schrodinger, Naturwissenschaften 23, 807 (1935).

[4] V. Vedral, M.B. Plenio, M.A. Rippin and P.L Knight, Phys. Rev. Lett. 78, 2275 (1997).

[5] E. Shchukin and W. Vogel, Phys. Rev. Lett. 95, 230502 (2005).

[6] A. Tal and G. Kurizki, Phys. Rev. Lett. 94, 160503, (2005)

[7] H. Mack and M. Freyberger, Phys. Rev. A 66, 042113 (2002).

[8] M.V. Fedorov et al., Phys. Rev. A 69, 052117 (2004).

[9] J. Eisert and M.B. Plenio, Int. J. Quant. Inf 1, 479 (2003).

[10] Tzu-Chieh Wei et al., Phys. Rev. A 67, 022110 (2003).

[11] A. Bertoni, J.Comp.Elec. 2, 291 (2003).

[12] P. Bordone and A. Bertoni, J.Comp.Elec 3, 407 (2004).

[13] D. Gunlycke, J.H. Jefferson, T. Rejec, A. Ramsak, D.G. Pettifor, G.A.D. Briggs, cond-matter/0509010.

[14] H. Mack and M. Freyberger, Phys. Rev. A 66, 042113 (2002).

[15] Yu Shi, Phys. Rev. A, 67, 024301 (2003).

[16] Y.S. Li, B. Zeng, X.S. Liu and G.L. Long, Phys. Rev. A, 64, 054302 (2001).

[17] P. Zanardi, Phys. Rev. A 65, 042101(R)(2002).

[18] J. Schliemann, J.I. Cirac, M. Kus, M. Lewenstein and D. Loss, Phys. Rev. A 64, 022303 (2001).

[19] J.R. Gittings and A.J. Fisher, Phys. Rev. A 66, 032305 (2002).

[20] R. Paskauskas and L. You, Phys. Rev. A 64, 042310 (2001).

[21] H.M. Wiseman and J.A. Vaccaro, Phys. Rev. Lett. 91, 097902 (2003).

[22] P. Zanardi and Wang X, J. Phys. A: Math. Gen. 35, 7947 (2002).

[23] Fernando G.S.L. Brandao, Phys. Rev. A 72, 022310 (2005).

[24] J. Schliemann, D. Loss, and A.H. MacDonald, Phys. Rev. B 63, 085311 (2001). 
[25] G.C. Ghirardi and L. Marinatto, Phys. Rev. A 70, 012109 (2004).

[26] X. Wang and B. Sanders, J. Phys. A: Math. Gen. 38, 67 (2005).

[27] K. Eckert, J. Schliemann, D. Bruss and M. Lewenstein, Annals of Physics 299, 88 (2002).

[28] M.L. Metha, Elements of Matrix Theory (Hindustian Publishing Corporation, Delhi,1977.)

[29] G. Ghirardi, L. Marinatto and T. Webber, J. Stat. Phys 108, 49 (2002).

[30] In order to scale the time of computation and to have a memory space big enough to allocate the matrices involved in the calculations, we have developed a parallel algorithm for matrix diagonalization. 